ORIGINAL ARTICLE

\title{
Simplifying thrombolysis decisions in patients with left bundle branch block
}

\author{
A D Reuben, C J Mann
}

Emerg Med J 2005;22:617-620. doi: 10.1136/emj.2003.013987

See end of article for authors' affiliations

......................

Correspondence to: Dr Adam Reuben,

Emergency Department, Royal Devon and Exeter Hospital, Barrack Road, Exeter, UK; adamreuben@ hotmail.com

Accepted for publication 18 August 2004

\begin{abstract}
Objectives: To redesign and simplify an existing decision algorithm for the management of patients who present to the emergency department with chest pain and left bundle branch block (LBBB) based on the Sgarbossa criteria. To compare its reliability with the current algorithm.

Methods: A simplified algorithm was created and tested against the existing algorithm. Electrocardiograms (ECGs) of patients with LBBB were presented to 10 emergency department doctors with both old and new algorithms a week apart. Six ECGs displayed the relevant criteria for thrombolysis and had proven acute myocardial infarction (AMI) based on a gold standard of enzyme measurements. Subjects were asked whether or not they would thrombolyse a patient presenting with the given ECG using each of the algorithms as a guide.

Results: The new algorithm has demonstrated improvements in terms of an increase in appropriate thrombolysis and a reduction in inappropriate thrombolysis. Specificity for AMI rose from 0.85 to 0.99 and sensitivity from 0.38 to 0.6 . $\kappa$ score showed greater agreement with the gold standard.

Conclusion: Patients with AMI and LBBB have a significantly poorer outcome than those without LBBB. Despite this, thrombolysis is less likely to be given to patients with AMI and LBBB. This study demonstrates that in part this is because of cognitive difficulties using the current algorithm. The proposed proforma addresses these issues and provides a simple tool to aid appropriate treatment in this group of patients.
\end{abstract}

$T$ he electrocardiogram (ECG) features of myocardial infarction (MI) are notoriously difficult to identify in the presence of left bundle branch block (LBBB). ${ }^{12}$ Consequently this has led to a reluctance or delay in the provision of thrombolysis to patients falling into this category. The beneficial effects of thrombolytic agents have long been known. ${ }^{3}$ Patients with MI and LBBB on their presenting ECG form an important group, having amongst the highest mortality of all patients with MI but also having the greatest benefit from thrombolytic treatment. ${ }^{4-6}$ Difficulty in interpreting the ECG of such patients can compromise their care.

Over the past decade various strategies to identify ECG characteristics of LBBB associated with acute myocardial infarction (AMI) have been proposed..$^{7-10}$ Most of these have been unsuccessful, proving far too complex and difficult to interpret and apply, and making an already confusing situation worse. ${ }^{91-13}$ One set of criteria that has gained a reasonable amount of support was proposed by Sgarbossa and colleagues (fig 1). ${ }^{14}$ They reviewed over 26000 ECGs of patients enrolled in the GUSTOI trial who had LBBB and MI confirmed on enzyme measurements and from these they devised three criteria that they found to have a high association with MI. They validated their criteria and their usefulness has been supported by several studies subsequently. ${ }^{15}$ Anecdotal evidence suggests that as useful as the criteria are, they are under used in clinical practice and this may be due in part to the perceived difficulty in application of the concepts of concordant and discordant ST segment elevation. ${ }^{12}{ }^{11} 16$ Understandably, there remains a reluctance to expose patients to the potentially fatal risks of cerebrovascular haemorrhage given that a proportion of those with chest pain and LBBB are not infarcting. ${ }^{1}$ Figure 2 shows an ECG with LBBB displaying Sgarbossa criteria for thrombolysis.

This study was undertaken to simplify the situation about the thrombolysis of patients with chest pain and LBBB.

\section{METHODS}

The three Sgarbossa criteria previously identified as having a high association with MI in patients with LBBB were reworded and simplified. The terms concordant and discordant ST segment elevation previously highlighted as causing the greatest confusion were removed. Based on these redefined criteria a simple flow treatment algorithm was created (fig 3).

An ECG database was compiled from archives of patients presenting to the emergency department with chest pain and LBBB. From a pool of ECGs, 17 were selected for final presentation. Six of these were of patients who had been subsequently shown to have proven AMI (based on troponin and creatinine-kinase measurements) and also displayed some or all of the Sgarbossa criteria. The other 11 ECGs were from patients in whom a MI was excluded and none of the criteria were present.

The ECGs were presented to 10 emergency department Senior House Officers with a copy of the original Sgarbossa algorithm. Half the doctors were given the current algorithm to aid their interpretation and the other half the redesigned proforma. Each was asked to decide, on the basis only of the algorithm presented to them, whether or not they would thrombolyse a patient presenting with each ECG, given a history of chest pain and no contraindications. There was no preceding educational session about the intricacies of the application of the Sgarbossa criteria. The process was repeated a fortnight later, with each group using the alternative algorithm and without any exposure to either algorithm in the interim.

Responses were evaluated and subjected to statistical analysis.

Abbreviations: $A M I$, acute myocardial infarction; $E C G$, electrocardiogram; LBBB, left bundle branch block; MI, myocardial infarction 


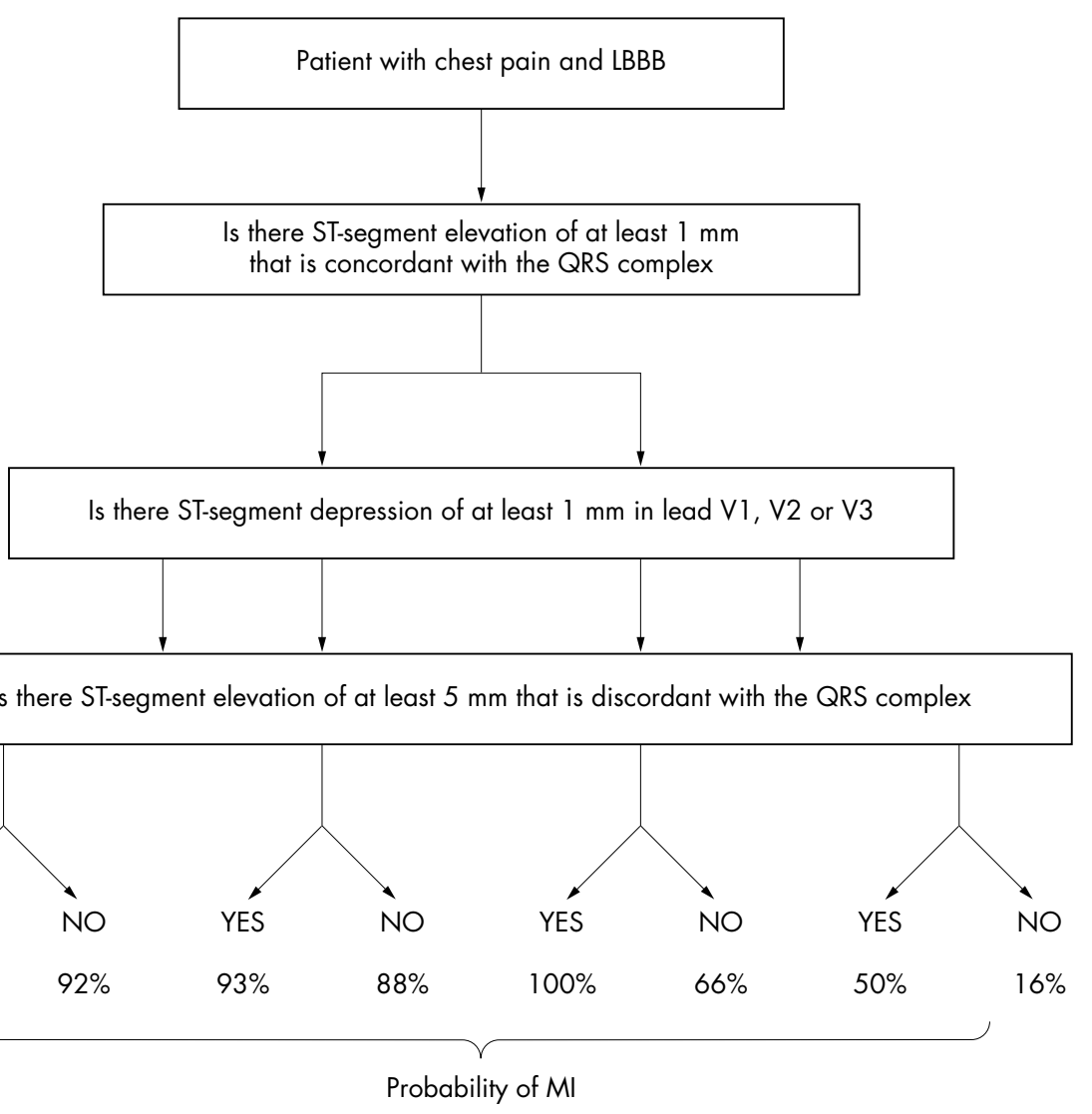

Figure 1 Original flow chart for the prediction of acute myocardial infarction in the presence of left bundle branch block (LBBB)

\section{RESULTS}

Ten emergency department SHOs reviewed the 17 selected ECGs and the appropriate data were accumulated following the 2 week collection period. Table 1 shows the number of candidates correct in assessing each case as being an MI or not. The mean number of correct diagnoses with the old algorithm was 6.8 compared with 8.5 using the modified algorithm. Application of the Wilcoxon test reveals that the number of candidates getting a correct diagnosis- that is, correct use of thrombolysis-was significantly greater with the new algorithm $(p=0.006)$. $\kappa$ Coefficients demonstrated better levels of agreement with the gold standard using the new algorithm compared with the old algorithm (table 2). Table 3 shows the sensitivity and specificity (\%) comparing each candidate's assessments with the gold standard for the 17 cases. Overall sensitivity increased from $38-60 \%$, whereas specificity rose from $85-99 \%$.

\section{DISCUSSION}

The benefits of thrombolysis in patients with acute MI in the presence of LBBB are well documented. ${ }^{3}$ Patients with LBBB have amongst the highest mortality of all patients with MI and also have the greatest potential benefit from thrombolysis. ${ }^{4}$ Despite this, anecdotal evidence suggests that thrombolytic agents are still under used in patients with LBBB. ${ }^{17}$ This may reflect a reluctance to administer potentially harmful agents to patients who may not be infarcting or may be because of difficulties arising from the interpretation of current criteria. ${ }^{18}$

The Sgarbossa criteria and algorithm were developed as a solution. For algorithms to be of value, it is essential that they are easily followed and simple to apply in a practical situation. Anecdotal evidence suggests that although the criteria do have a role to play in the evaluation of ECGs showing LBBB, they are both difficult to apply and to

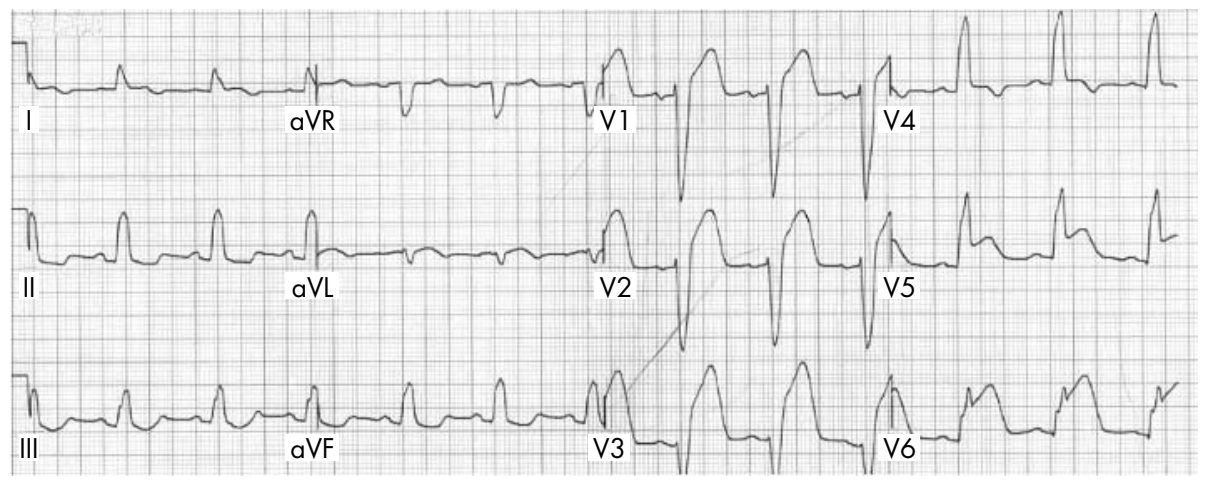

Figure 2 Electrocardiogram demonstrating Sgarbossa criteria for thrombolysis - ST elevation $>5 \mathrm{~mm}$ in leads V1, V2, V3, and ST elevation $>1 \mathrm{~mm}$ in leads $\mathrm{V} 5, \mathrm{~V} 6$. 


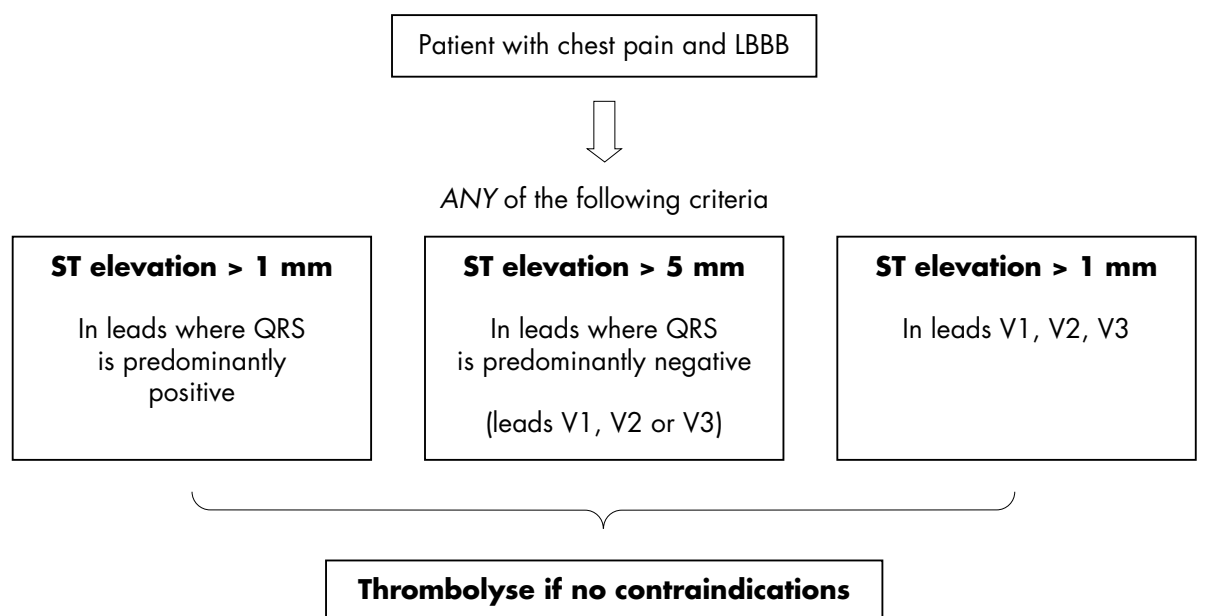

Figure 3 Revised flow chart for the management of patients with chest pain in the presence of left bundle branch block (LBBB)

interpret and are therefore underused. We endeavoured to create a new algorithm for the management of patients presenting with chest pain and LBBB, based upon the criteria described and validated by Sgarbossa, but one which would be easier to interpret and to apply and thus lead to a greater utilisation.

The original Sgarbossa algorithm incorporated a weighting system for their criteria so that each was associated with a different probability of MI. ${ }^{14}$ This in part contributed to the complexity of the algorithm. Subsequent work has shown that the presence of any of the three predictive electrographic criteria had a high association with $\mathrm{MI}^{-1}$ therefore, we have given each of the criteria an equal weighting in our new algorithm and thus eliminated the complexities associated with differential weighting and increased the sensitivity of the algorithm. It is important to recognise that there are inherent limitations associated with the Sgarbossa criteria and these are reproduced in the new algorithm. In the absence of any of the three criteria, there remains a significant possibility of myocardial ischaemia. However, the aim of the redesigned algorithm is to facilitate easier application of the existing criteria and therefore improve the recognition and thrombolysis of patients displaying them.

\begin{tabular}{|c|c|c|}
\hline Case & Old & New \\
\hline 1 & 5 & 8 \\
\hline 2 & 5 & 9 \\
\hline 3 & 7 & 10 \\
\hline 4 & 9 & 10 \\
\hline 5 & 3 & 9 \\
\hline 6 & 10 & 10 \\
\hline 7 & 8 & 8 \\
\hline 8 & 9 & 10 \\
\hline 9 & 9 & 10 \\
\hline 10 & 10 & 10 \\
\hline 11 & 9 & 10 \\
\hline 12 & 4 & 7 \\
\hline 13 & 9 & 10 \\
\hline 14 & 1 & 3 \\
\hline 15 & 9 & 10 \\
\hline 16 & 7 & 10 \\
\hline 17 & 2 & 1 \\
\hline Mean & 6.8 & 8.5 \\
\hline Median & 8 & 10 \\
\hline
\end{tabular}

We have demonstrated, using our new algorithm, a higher rate of correct application of the Sgarbossa criteria, which in practical terms would correspond to a higher rate of thrombolysis in patients with LBBB. We have further demonstrated that the new algorithm has higher sensitivity and specificity for detecting acute MI in our sample. The $\kappa$ scores demonstrate a good level of interobserver agreement with the gold standard.

It is likely that this will remain a controversial area. The "thrombolyse all" and "thrombolyse some" approaches both have strong advocates. ${ }^{111} 14^{16}{ }^{18-20}$ Shlipak and colleagues

Table $2 \kappa$ Coefficients ( $p$ values) for comparing each candidate's assessments with gold standard for the 17 cases

\begin{tabular}{lll}
\hline Candidate & Old & New \\
\hline 1 & $0.39(0.04)$ & $0.87(<0.001)$ \\
2 & $-0.12(0.58)$ & $0.56(0.01)$ \\
3 & $0.0(1.0)$ & $0.72(0.002)$ \\
4 & $0.48(0.05)$ & $0.56(0.01)$ \\
5 & $0.39(0.04)$ & $0.56(0.01)$ \\
6 & $0.44(0.06)$ & $0.56(0.01)$ \\
7 & $0.06(0.79)$ & $0.44(0.06)$ \\
8 & $0.16(0.48)$ & $0.72(0.002)$ \\
9 & $0.27(0.21)$ & $0.72(0.002)$ \\
10 & $0.44(0.06)$ & $0.72(0.002)$ \\
\hline
\end{tabular}

Table 3 Sensitivity and specificity (\%) for comparing each candidate's assessments with gold standard for the 17 cases

\begin{tabular}{clrlll}
\hline & \multicolumn{3}{l}{ Old } & & New \\
\cline { 2 - 3 } \cline { 5 - 5 } Candidate & Sensitivity & Specificity & & Sensitivity & Specificity \\
\hline 1 & 33 & 100 & 83 & 100 \\
2 & 50 & 36 & 50 & 100 \\
3 & 0 & 100 & 67 & 100 \\
4 & 67 & 82 & 50 & 100 \\
5 & 33 & 100 & 50 & 100 \\
6 & 50 & 91 & 50 & 100 \\
7 & 33 & 73 & 50 & 91 \\
8 & 33 & 82 & 67 & 100 \\
9 & 33 & 91 & 67 & 100 \\
10 & 50 & 91 & 67 & 100 \\
Mean & 38 & 85 & 60 & 99 \\
\hline
\end{tabular}


carried out a decision analysis using a retrospective cohort study to which a decision-based analysis was applied. ${ }^{11}$ They calculated that among 1000 patients with LBBB and chest pain, more would survive without major haemorrhage if all received thrombolysis than if the Sgarbossa ECG algorithm was used as a screening test. They concluded that the ECG is a poor predictor of MI in patients with LBBB and acute cardiopulmonary symptoms and therefore acute thrombolytic treatment should be considered for all patients with LBBB who have symptoms consistent with MI. The low sensitivity of the ECG criteria may be explained by idiosyncratic characteristics of the studied population and the benefits of thrombolysis may have been amplified by assumptions in the decision analysis. However, this is still the course of action currently recommended by the American Cardiac Congress and the American Heart Association. ${ }^{19}$

Li et al carried out a retrospective cohort study in an attempt to validate the Sgarbossa criteria. ${ }^{2}$ They reviewed the ECGs of 190 patients who had presented with chest pain and LBBB on the initial ECG. Twenty five patients (13\%) had MI confirmed based on CK-MB measurement. Although they found the criteria to be very specific for MI (93-100\%), their sensitivities for detection were low $(0-16 \%)$ giving a poor negative likelihood ratio. They concluded from this study that the criteria cannot be used to exclude MI in patients with LBBB. However, the authors recognised that although the criteria were unsuitable for exclusion of MI, their high specificity meant that when they are present there was a very high association with MI and thus a good case is presented for thrombolytic treatment in the absence of contraindications.

Clearly the situation remains complex. A "thrombolysis for all" approach is difficult to justify considering the potentially large proportion of infarct-free patients. ${ }^{1}$ Until we have the facilities for primary angioplasty for all patients with suspected acute infarction, difficult decisions about the thrombolysis of patients with LBBB will undoubtedly have to be taken. Until such a time, we must rely upon the resources currently available to us, and thrombolysis of those patients who are deemed to be at highest risk of infarction on the basis of a good history and examination findings would seem sensible and in keeping with current practice in many departments. $^{21}$

Low sensitivities mean that the Sgarbossa criteria are of little value as a "rule out" tool for AMI as they are all too often absent in the presence of infarction. ${ }^{21}$ This does not mean that they do not have a role to play in the diagnostic process. The criteria are highly specific and can be reliably used as a confirmatory test to "rule in" AMI in patients with LBBB. $^{20}$ Although not the ideal solution to the problem of the management of patients who present with chest pain and LBBB, the Sgarbossa criteria ensure that at least some of the patients with AMI will receive thrombolysis. The results of our limited study suggest that the new algorithm improves the application of the Sgarbossa criteria and could thus lead to the safe, appropriate, thrombolysis of more patients. More work on a larger scale will be needed to confirm this benefit.

\section{Authors' affiliations}

A D Reuben, A\&E, Musgrove Park Hospital, Taunton, Exeter, UK C J Mann, A\&E, Taunton \& Somerset Hospital, Parkfield Drive, Taunton, UK

Competing interests: none declared

\section{REFERENCES}

1 Edhouse JA, Sakr M, Angus J, et al. Suspected myocardial infarction and left bundle branch block: electrographic indicators of acute ischaemia. J Accid Emerg Med 1999;16:331-5.

2 Li SF, Walden PL, Marcilla O, et al. Electrocardiographic diagnosis of myocardial infarction in patients with left bundle branch block. Ann Emerg Med 2000;36:561-5.

3 ISIS-2 Collaborative Group. Randomised controlled trial of intravenous streptokinase, oral aspirin, both, or neither among 17,187 cases of acute myocardial infarction: ISIS-2. Lancet 1988;2:349-60.

4 Fibrinolytic Therapy Trialists' (FTT) Collaborative Group. Indications for fibrinolytic therapy in suspected acute myocardial infarction: collaborative overview of early mortality and major morbidity results from all randomised trials with more than 1000 patients. Lancet 1994;343:311-22.

5 Newby KH, Pisano E, Krukoff MW, et al. Incidence and clinical relevance of the occurrence of bundle-branch block in patients treated with thrombolytic therapy. Circulation 1996;94:2424-8.

6 Hindman MC, Wagner GS, Jaro M, et al. The clinical significance of bundle branch block complicating acute myocardial infarction. Circulation 1978:58:679-88.

7 Besoain-Santander M, Gomez-Ebensperguer G. Electrocardiographic diagnosis of myocardial infarction in cases of complete left bundle branch block. Am Heart J 1960;60:886-97.

8 Weiner R, Makam S, Gooch AS. Identification of myocardial infarction in the presence of left bundle branch block: correlation of electrocardiography, vectorcardiography and angiography. J Am Osteopath Assoc 1983;83:119-24.

9 Wackers FJ. The diagnosis of myocardial infarction in the presence of left bundle branch block. Cardiol Clin 1987;5:393-401.

10 Hands ME, Cook EF, Stone PH. Electrocardiographic diagnosis of complete myocardial infarction in the presence of left bundle branch block. Am Heart $J$ 1988;116:23-31.

11 Shlipak MG, Lyons WL, Go AS, et al. Should the electrocardiogram be used to guide therapy for patients with left bundle branch block and suspected myocardial infarction? JAMA 1999;281:714-19.

12 Wackers FJ. Complete left bundle branch block: is the diagnosis of myocardial infarction possible? Int J Cardiol 1983;2:521-9.

13 Pryor R. Recognition of myocardial infarction in the presence of bundle branch block. Cardiovasc Clin 1974;6:255-71.

14 Sgarbossa EB, Pinski SL, Barbagelata A, et al. Electrocardiographic diagnosis of evolving myocardial infarction in the presence of left bundle branch block. N Engl J Med 1996;334:481-7.

15 Madias JE, Sinha A, Ashtlani R, et al. A critique of the new ST-segment criteria for the diagnosis of acute myocardial infarction in patients with left bundle branch block. Clin Cardiol 2001;24:652-5.

16 Gallagher JE. Editorial: Which patients with suspected myocardial ischaemia and left bundle branch block should receive thrombolytic agents? Ann Emerg Med 2001;37:439-44.

17 Barron HV, Bowlby $\amalg$, Breen T, et al. Use of reperfusion therapy for acute myocardial infarction in the United States. Data from the national registry of myocardial infarction 2. Circulation 1992;97:1150-6.

18 Maggioni AP, Franzosi MP, Santoro E, et al. The risk of stroke in patients with acute myocardial infarction after thrombolytic and antithrombotic treatment. N Engl J Med 1992;327:1-6.

19 Ryan TJ, Antman EM, Brooks NH, et al. ACC/AHA guidelines for the management of patients with acute myocardial infarction: 199 update. Available at: http://www.acc.org/clinical/topic/topic.htm and http:// www.americanheart.org (last accessed 29 June 05).

20 Kontos MC, McQueen RH, Jesse RL, et al. Can myocardial infarction be rapidly identified in emergency department patients who have left bundle branch block? Ann Emerg Med 2001;37:431-8.

21 Wellens HJ. Acute myocardial infarction and left bundle branch block-can we lift the veil? N Engl J Med 1996;334:528-9. 


\section{PostScript}

\section{CORRECTIONS}

doi: 10.1136/emj.2003.13607corr 1

$\mathrm{n}$ the Emergency casebook titled, Delayed presentation of internal mammary artery rupture after blunt chest trauma: characteristic CT and plan x ray findings (Emerg Med $J$ 2005;22:664-5), the author affiliations were incorrect. The correct affiliations are as follows:

S P Chung, I S Yoo, S W Kim Department of Emergency Medicine, Chungnam National University Hospital, Daejeon, Republic of Korea
O Y Kwon Department of Anatomy, College of Medicine, Chungnam National University, Daejeon, Republic of Korea

C J Song Department of Radiology, Chungnam University Hospital, Daejon, Republic of Korea

I B Kim Department of Emergency Medicine, Kwandong University College of Medicine, Myongji Hospital, Kyunggi-do, Republic of Korea

doi: 10.1136/emj.2003.12914corrl

n the letter titled, Difficult adherent nail bed dressings: An escape route (Emerg Med $J$ 2005:22:312), the lead authors name is incorrectly cited as M S Ul Hassan. The correct citation is M S U Hassan. The journal apologises for this error.

doi: 10.1136/emj.2003.13987corr 1

n error has occurred in figure 3 of the paper titled, Simplifying thrombolysis decisions in patients with left bundle branch block (Emerg Med J 2005;22:617-20). The third box from the left should read ST depression $>1 \mathrm{~mm}$ instead of ST elevation $>$ $1 \mathrm{~mm}$. Please see correct figure 3 below.

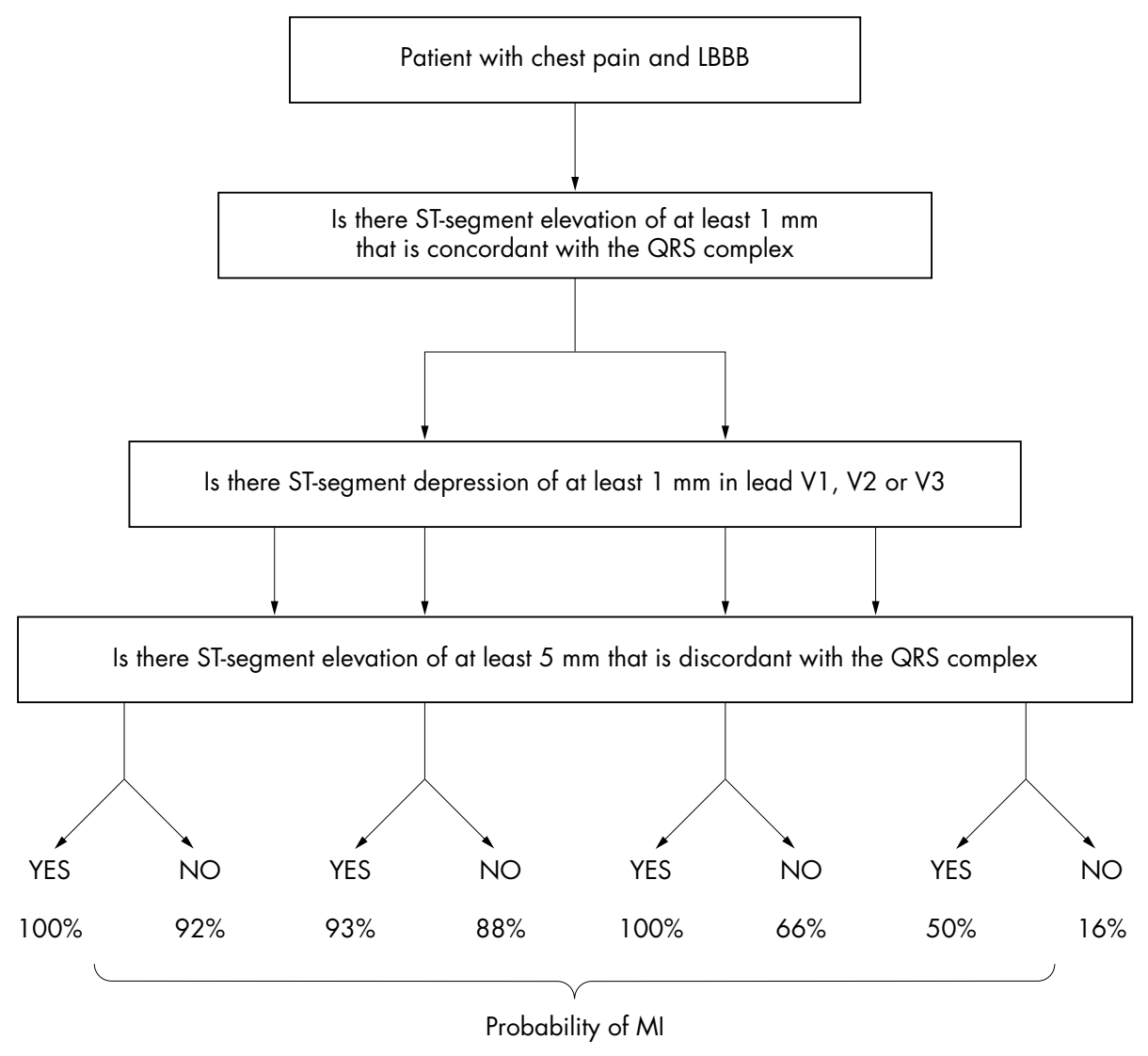




\section{CORRECTIONS}

doi: 10.1136/emj.2005.25346corr l

The author affiliations and correspondence details are incorrect for the paper titled, Use of a forensic technique to identify blood contamination of emergency department and ambulance trauma equipment (Emerg Med J 2006;23:73-5). The correct author affiliations are:

JB Lee, M Levy, Yorkshire Deanery UK

A Walker, Mid-Yorkshire Hospital Trust, UK

Correspondence should be sent to: Miss A Walker, Mid-Yorkshire Hospitals Trust, UK; alison.walker1@midyorks.nhs.uk.

doi: 10.1136/emj.2003.13987corr2

Thrombolyse if no contraindications

An error occurred in figure 3 of the paper titled, Simplifying thrombolysis decisions in patients with left bundle branch block (Emerg Med J 2005;22:617-20). The corrections printed in the November issue (Emerg Med $J$ 2005;22:836) was also incorrect. Please find below the correct figure. The journal apologises for this error.

Patient with chest pain and LBBB

ANY of the following criteria

\begin{tabular}{|c|c|}
\hline $\begin{array}{c}\text { ST elevation > } 1 \text { mm } \\
\text { In leads where QRS } \\
\text { is predominantly } \\
\text { positive }\end{array}$ & $\begin{array}{c}\text { ST elevation > } 5 \mathbf{~ m m} \\
\text { In leads where QRS } \\
\text { is predominantly negative } \\
\text { (leads V1, V2 or V3) }\end{array}$ \\
\hline
\end{tabular}

ST depression > $1 \mathrm{~mm}$

In leads V1, V2, V3 